Reprod. Nutr. Dévelop., 1986, 26 (1 B), 359-360.

\title{
Influence de l'état corporel au vêlage et du niveau d'alimentation en début de lactation sur la stéatose hépatique chez la vache laitière
}

\author{
Y. RAYSSIGUIER, A. MAZUR, B. REMOND, Y. CHILLIARD, Elyett GUEUX \\ I.N.R.A., Theix, 63122 Ceyrat, France.
}

Summary. Improving body status at calving resulted in a significant increase of the liver triglyceride levels in dairy cows at the onset of lactation and particulary in feed-restricted animals.

La lipomobilisation chez les vaches laitières haute productrices en début de lactation est à l'origine d'une infiltration lipidique du foie qui pourrait être conditionnée en grande partie par l'état nutritionnel.

Matériel et méthodes. Nous avons voulu étudier l'interaction entre l'état corporel et le niveau d'alimentation au début de la lactation chez 43 vaches PieNoires ayant un potentiel laitier d'environ $30 \mathrm{~kg} / \mathrm{j}$ et recevant un régime à base d'ensilage de maïs. Les animaux étaient répartis en 4 groupes expérimentaux faisant intervenir deux états corporels au vêlage (gras : $G$ et maigre : $M$, correspondant à des notes de 3,9 et $1,8 / 5)$ combinés à deux niveaux d'alimentation après le vêlage : haut $(\mathrm{H})$ et bas $(\mathrm{B})$ correspondant à une alimentation ad libitum ou restreinte de $1,7 \mathrm{~kg} \mathrm{MS} /$ jour pendant la semaine qui a précédé les prélèvements (Rémond et Chilliard, non publié). Un prélèvement sanguin a été effectué, 12 jours après le vêlage, le matin à jeun et suivi d'une biopsie hépatique d'une à deux heures après la distribution de l'aliment. Des dosages enzymatiques ont permis d'évaluer les teneurs en acides gras libres (AGL), glucose, $\beta$-hydroxybutyrate $(\mathrm{BHB})$ du plasma et les teneurs en triglycérides du foie.

Résultats et discussion. Le taux de triglycérides hépatiques est significativement plus élevé chez les vaches grasses que chez les vaches maigres, quel que soit le niveau d'alimentation après le vêlage. Les taux d'AGL et de BHB plasmatique sont essentiellement influencés par le niveau d'alimentation après le vêlage, une alimentation restreinte entraînant leur augmentation. Ce phénomène n'est significatif que chez les vaches grasses mais une tendance identique s'observe également chez les vaches maigres (tabl. 1). Il existe donc une certaine dissociation entre les paramètres témoins de la lipomobilisation ou de la cétogenèse, et le niveau d'infiltration lipidique du foie.

Les animaux ont été classés en fonction de leur teneur hépatique en triglycérides: vaches normales, vaches à stéatose modérée ou à stéatose sévère, pour des valeurs respectivement inférieures à $50 \mathrm{mg} / \mathrm{g}$, comprises entre 50 et $100 \mathrm{mg} / \mathrm{g}$, et supérieures à $100 \mathrm{mg} / \mathrm{g}$ de poids frais. Globalement, un état d'engraissement excessif au moment du vêlage favorise l'apparition de la stéatose, contrairement au niveau alimentaire après le vêlage dont l'influence n'est pas significative. II y a toutefois une interaction entre les 2 facteurs puisque la fréquence est significativement accrue pour le lot $\mathrm{G} B$ et significativement diminuée 
pour le lot $\mathrm{MH}$, le nombre de cas de stéatose sévère étant particulièrement important dans le lot G B. Les animaux stéatosiques montrent des taux d'AGL et de $B H B$ significativement plus élevés et une glycémie significativement plus faible (tabl. 2).

TABL. 1. - Influence de l'état corporel au vêlage ( $\mathrm{G}$ et $\mathrm{M}$ ) et du niveau d'alimentation (H et $\mathrm{B}$ ) en début de lactation, sur les différents paramètres plasmatiques et l'infiltration lipidique du foie.

\begin{tabular}{lcccc}
\hline Régime alimentaire & $\mathrm{G} \mathrm{H}$ & $\mathrm{G} \mathrm{B}$ & $\mathrm{M} \mathrm{H}$ & $\mathrm{M} \mathrm{B}$ \\
\hline AGL (mM) & $0,63 \pm 0,11^{\mathrm{a}}$ & $1,17 \pm 0,19^{\mathrm{b}}$ & $0,46 \pm 0,08^{\mathrm{a}}$ & $0,66 \pm 0,08^{\mathrm{a}}$ \\
Glucose (mM) & $3,11 \pm 0,17^{\mathrm{a}}$ & $2,89 \pm 0,22^{\mathrm{a}}$ & $3,22 \pm 0,17^{\mathrm{a}}$ & $3,11 \pm 0,11^{\mathrm{a}}$ \\
BHB (mM) & $1,29 \pm 0,18^{\mathrm{a}}$ & $2,20 \pm 0,42^{\mathrm{b}}$ & $1,08 \pm 0,18^{\mathrm{a}}$ & $1,90 \pm 0,37^{\mathrm{ab}}$ \\
$\begin{array}{l}\text { Triglycérides du foie } \\
\text { (mg/g poids frais) }\end{array}$ & $80 \pm 20^{\mathrm{a}}$ & $90 \pm 17^{\mathrm{a}}$ & $26 \pm 8^{\mathrm{b}}$ & $33 \pm 7^{\mathrm{b}}$ \\
\hline $\begin{array}{l}\text { Nombre d'animaux } \\
\text { Vaches avec stéatose }\end{array}$ & 11 & 9 & 12 & 11 \\
sévère* & 2 & 4 & 0 & 0 \\
$\begin{array}{l}\text { Vaches avec stéatose } \\
\text { modérée* }\end{array}$ & 4 & 3 & 2 & 4 \\
\hline
\end{tabular}

Moyenne \pm erreur type. Les différences significatives $(P<0,05)$ entre moyennes sont indiquées par des lettres différentes. * Le nombre de vaches stéatosiques dans chaque lot expérimental est influencé significativement par l'état d'engraissement au moment du vêlage $\left(G, M \chi^{2}=6,57 ; P=0,01\right)$. L'influence de l'alimentation après le vêlage n'est pas significative $\left\langle H, B \chi^{2}=1,77\right)$

TABL. 2. - Relation entre différents paramètres plasmatiques et la stéatose hépatique.

\begin{tabular}{lccc}
\hline (nombre d'animaux) & $\begin{array}{c}\text { Normales } \\
(22)\end{array}$ & $\begin{array}{c}\text { Stéatose } \\
\text { modérée } \\
(13)\end{array}$ & $\begin{array}{c}\text { Stéatose } \\
\text { sévère } \\
(6)\end{array}$ \\
\hline $\begin{array}{l}\text { Triglycérides du foie } \\
\text { (mg/g poids frais) }\end{array}$ & $20 \pm 2$ & $74 \pm 5$ & $155 \pm 20$ \\
Glucose (mM) & $3,33 \pm 0,06^{\mathrm{a}}$ & $2,83 \pm 0,17^{\mathrm{b}}$ & $2,83 \pm 0,22^{\mathrm{b}}$ \\
BHB (mM) & $1,11 \pm 0,15^{\mathrm{a}}$ & $2,26 \pm 0,29^{\mathrm{b}}$ & $1,87 \pm 0,43^{\mathrm{b}}$ \\
AGL (mM) & $0,49 \pm 0,05^{\mathrm{a}}$ & $0,80 \pm 0,10^{\mathrm{b}}$ & $1,30 \pm 0,21^{\mathrm{c}}$ \\
\hline
\end{tabular}

En conclusion, cette expérimentation souligne l'importance de l'état corporel au moment du vêlage sur l'apparition de la stéatose hépatique au début de la lactation, les animaux gras présentant un risque accru par suite d'une lipomobilisation intense, les animaux maigres un risque diminué même si leur alimentation est restreinte par la suite (Reid, Roberts et Baird, 1980 ; Treacher, Reid et Roberts, 1981). Les paramètres plasmatiques témoins de la lipomobilisation et de la cétogenèse ne permettent pas d'apprécier suffisamment le risque de stéatose en l'absence de la détermination directe des triglycérides hépatiques.

Reid I. M., Roberts C. J., Baird G. D., 1980. The effects of underfeeding during pregnacy and lactation on structure and chemistry of bovine liver and muscle. J. agric. Sci. Camb., 94, 239-245.

Treacher R. J., Reid I. M., Roberts C. J., 1981. The effect of body condition at calving on the development of fatty liver and metabolic disease (Abstr.). Anim. Prod., 32, 362. 